Proc. Indian Acad. Sci., Vol. 87 A (E \& P Sciences-4), No. 11, November 1978, pp. 193-200, (C) printed in India

\title{
Periodicity in rainfall at Madras
}

\author{
G K RANGARAJAN and K N RAO \\ Indian Institute of Geomagnetism, Bombay 400005 \\ MS received 23 May 1978; revised 18 July 1978
}

\begin{abstract}
Monthly rainfall data from Madras for the period 1813-1976 are spectrally analysed for detection of significant oscillations using the new data adaptive technique of maximum entropy developed by Burg. Spectra for annual, NE monsoon and SW monsoon rainfall are separately presented and compared with the spectra derived from Fourjer transform using FFT. The long series of data are divided into several subsets and analysed to determine the stability of the periodicities. The effect of changing the length of PEF on the resultant spectra is also discussed. The analysis reveals that longer period variations ( $>20$ years) in the rainfall is either absent or non-stationary. Solar-cycle periodicity of 11 years is also missing in the rainfall. A quasibiennial oscillation in the annual and monsoon rainfall is observed in all the subsets and in the full series. Stacked spectrum derived from the spectra of monthly rainfall series for 30-year spans is dominated by the annual line and its harmonics. The biennial pulse is absent in the monthly rainfall series.
\end{abstract}

Keywords. Rainfall; spectrum; maximum entropy method; periodicity.

\section{Introduction}

Among the many Indian stations that provide systematic rainfall measurements, the observatory in Madras City (Nungambakkam) has the unique distinction of the longest recorded series with an almost unchanged exposure factor. Data available from 1813 provide a homogeneous time series and, therefore, recent techniques of analysis for detection of significant oscillations in time series can be applied confidently to the rainfall at Madras. Trends and periodicities in rainfall at a number of Indian stations or in meteorological subdivisions have been studied adopting for analysis, numerical filters, orthogonal polynomials and/or power spectral analysis following the correlation-cosine transform procedure of Blackman and Tukey (1959). With the advent of fast Fourier transform (FFT) routines more reliable spectral estimates could be obtained in less time. However, both the methods suffer from the drawbacks associated with unrealistic assumption regarding extension of data outside the sample interval, specially in the case of FFT. Burg (1975) obviated the need for such an extension through a radically different approach to spectral analysis using the concept of entropy, called the Maximum Entropy Method (MEM). (For a detailed exposition Smylie et al 1973; Ulrych and Bishop 1975 and others may be seen). It has been shown by Burg (1975) that MEM is data adaptive, needs no windowing functions for smoothing, does not consider the nature of the data outside the sample and provides a stable smooth spectrum with high resolution. One of the major shortcomings of this method is no doubt, the choice of the optimal length 
(L) of the Prediction Error Filter (PEF). While several suggestions on the choice of the length have been made in recent years (for e.g. Ulrych and Bishop 1975) there has been no satisfactory solution to this problem yet. It is known that a small value of $L$ leads to a highly smoothed spectrum obviating the resolution advantage of MEM, whereas too large a value leads to instability and causes line splitting (Chen and Stegen 1974; Fougere et al 1976). It is usually recommended that the spectrum be computed for several values of $L$ and a suitable choice is then made that leads to a spectrum consistent with known characteristics of the time series. Courtillot et al (1977) recently concluded that a choice of $20 \%$ to $50 \%$ of the length of the data sample was correct in most cases. Upper limit of $50 \%$ was also suggested by Ulrych and Bishop (1975).

The monthly and annual rainfall of Madras (Nungambakkam) for the period 1813-1976 is examined for periodicity. For the period, September 1943 to December 1946, when there was loss of Nungambakkam data, data from the neighbouring observatory located at the airport were used for interpolation (vide Rao and Raghavendra 1973). The monthly data for October to December and June to September were combined as representative of the NE monsoon seasonal and SW monsoon seasonal rainfall of Madras respectively. These, together with the annual rainfall thus provide three time series with $\Delta t=1$ year.

\section{Method}

In view of the improved resolution capabilities and stability of the spectral estimates derived by the method of maximum entropy in contrast to FFT and BlackmanTukey approaches, we have used MEM to obtain the spectral composition of the three time series of rainfall. In a comparison of decade means of rainfall with the overall average, Rao and Raghavendra (1973) did not detect statistically significant difference and concluded that the Madras rainfall data did not show any trend. Hence we have not adopted any numerical procedure for elimination of trend in the three time series.

To study the effect of varying length of the PEF on the rainfall spectrum we computed the spectra for several lengths $(L)$ of the prediction error filter in the range 20 to $30 \%$ of the total data. We noticed that even at $30 \%$ the splitting of spectral lines occurred so that we did not go upto the upper limit of $50 \%$ recommended by Ulrych and Bishop (1975).

\section{Results and discussion}

In table 1 is summarised the periods corresponding to which a peak was noticed in the respective spectra and their amplitudes. In the spectra derived by BlackmanTukey or fast Fourier transform approaches, the ordinate at each frequency is directly proportional to the variance and, therefore, to the square of the amplitude of the oscillation. Experiments with MEM have clearly indicated that in the spectra computed with Burg's algorithm, the area under the spectral peak is the significant quantity (Lacoss 1971; Radoski et al 1975). The amplitudes presented in table 1 are obtained as the square root of the product of the power density and the band-width 


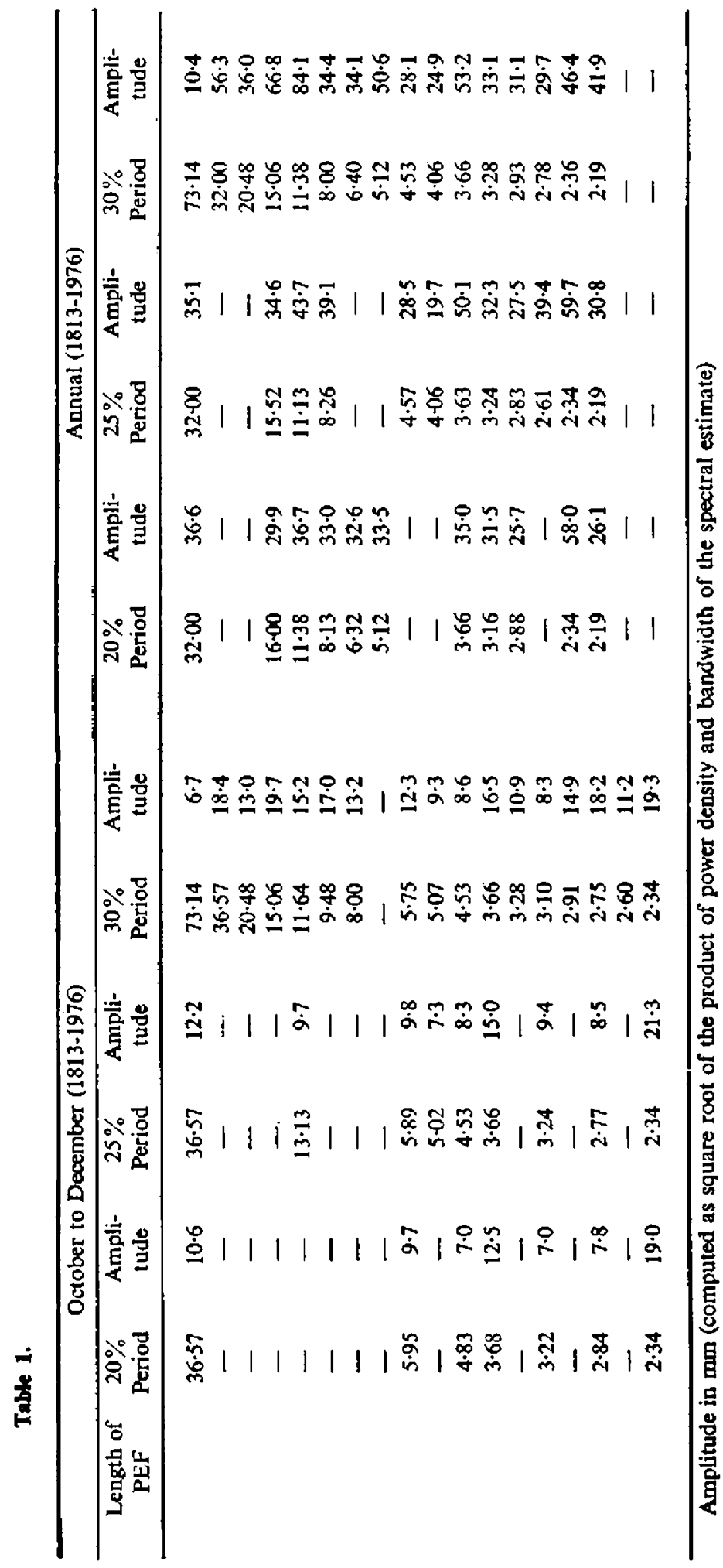


of the estimate. It is known that the amplitudes could be biased by a large factor (Currie 1975; Rangarajan and Arora 1977) but the relative amplitudes at different frequencies are reliable. The main advantage in the maximum entropy method of spectral analysis, of course, is its ability to isolate significant periodicities without undue frequency shift. No known statistical tests of significance, however, can be applied to the spectral peaks directly in view of the limitation mentioned above. It is seen that at $20 \%$ the characteristics of the spectrum are almost well determined for all the three time series since the periodicities detected adopting this length of filter weights are repeated in the spectra with longer lengths of the filter. In figure 1 are presented the spectra of the three series- 'Annual', October-December and JuneSeptember rainfall with $L=37$ (i.e. $22.5 \%$ ). Spectra of annual and NE monsoon rainfall data of Madras derived from FFT based on the algorithm of Cooley and Tukey (1965) following the details given by Rangarajan and Bhargava (1974) are also shown in figure 1 for comparison of the results by the two methods. While for identical bandwidth of the spectral estimates the Fourier spectra have more ragged appearance, the prominent spectral peaks are well resolved in both. In the low frequency range, where the wavelength is comparable to the length of available data, the spectral peaks observed in MEM spectra should be more reliable because in this range the FFT spectra are known to introduce frequency shifts (Ulrych 1972 and others).

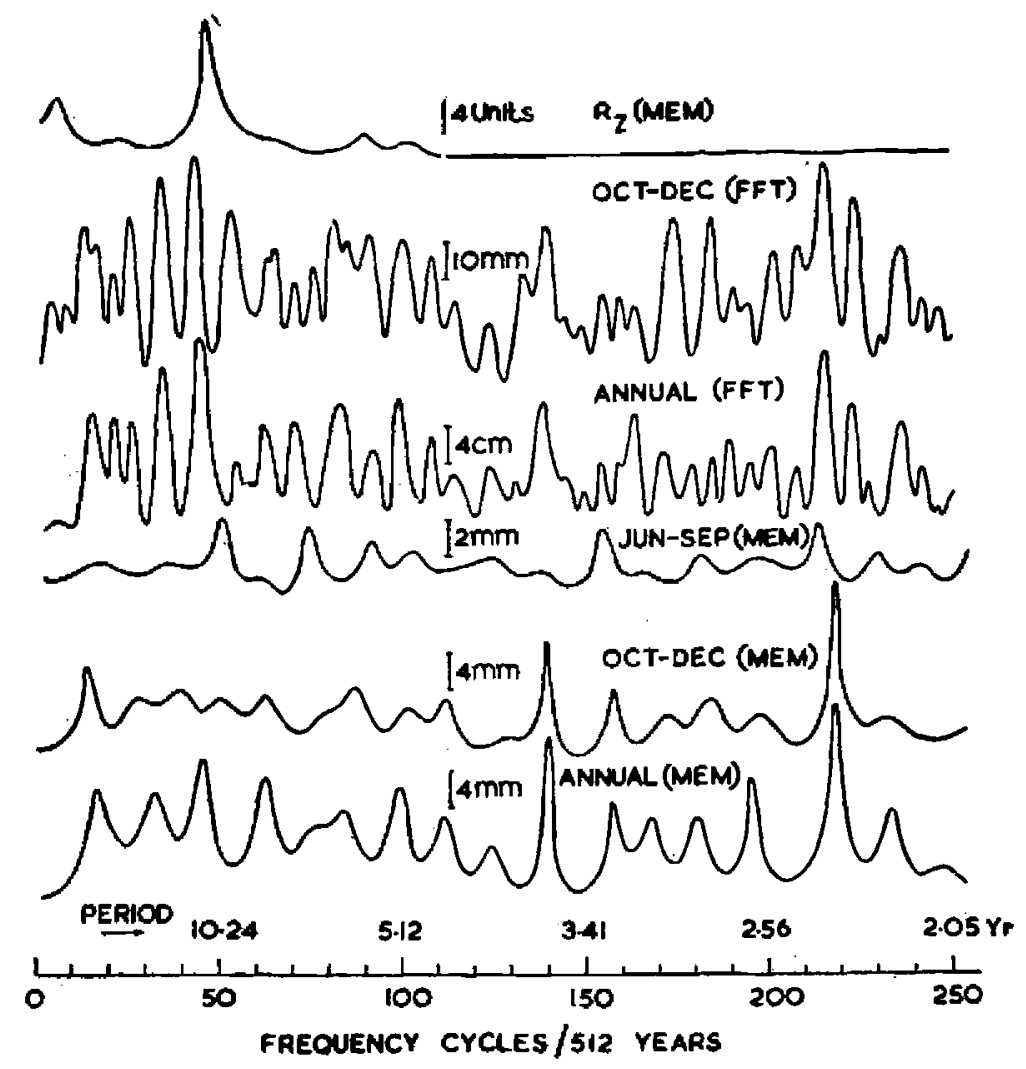

Figure 1. Amplitude spectrum of rainfall at Madras (1813-1976) derived by MEM and FFT. Spectrum (MEM) of annual mean sunspot number (Rz) for the same period is also shown for comparison. 
It is immediately apparent that there is no long-period variation ( $>40$ years) of any significance in the rainfall data. While the annual rainfall indicates a period of 32 years, in October-December series the corresponding nearest periodicity is at 36.6 years and no significant oscillation is seen in the June-September series, in the vicinity of this periodicity.

A feature of interest is that while June-September rainfall accounts for about onethird of the annual rainfall at Madras (Rao and Raghavendra 1973) the spectrum is not dominated by any strong periodic oscillation indicating that the rainfall during this period is quite stable in nature. In contrast, the annual rainfall spectrum indicates several significant periodicities, most striking of them being the ones corresponding to periods of 3.66 years and 2.34 years. A spectral peak corresponding to the sunspot cycle of 11 years is seen at 11.4 years but we shall see later that this is not stationary with time. In view of the fact that June-September rainfall spectrum is almost 'white' in nature, and since October-December rainfall is about $50 \%$ of the annual figure, it would be reasonable to expect a good degree of similarity between the spectra of October-December and annual rainfall. Figure 1 shows that it is indeed so with the 3.66 year and 2.34 year cycle dominating both the spectra. However, the annual rainfall spectrum in the low frequency range (period above 6 years) has a number of spectral peaks which are non-stationary, in nature and which do not have their counterpart in the NE monsoon data.

\section{Analysis of subsets}

In view of the fact that several factors contribute to the measure of rainfall during any span of time, it is likely that the time series could at best be quasi-stationary with only certain periodicities common to all cpochs. To test whether the three time series derived from rainfall data of Madras are stationary and to detect those periodicities which are stable, we analysed the rainfall data by dividing the total length into 5 blocks of about 81 years each as below:

$\begin{array}{rr}\text { I } & 1813-1893 \\ \text { II } & 1834-1914 \\ \text { III } & 1855-1935 \\ \text { IV } & 1876-1956 \\ \text { V } & 1897-1976\end{array}$

With the suitability of MEM to even short segments of data without loss of resolution and frequency shift even in the low frequency domain, we compute the spectra for the three time series for the 5 blocks of data adopting $25 \%$ as length of the prediction error filter.

Periods of spectral peaks prominent in relation to the continuum in the neighbourhood are listed in table 2.

For all the subsets, the June-September spectra are almost ' white' in nature and there is no significant periodicity common to all the 5 blocks except one that corresponds to Quasi Biennial Oscillation (QBO) varying in period from 2.3 to 2.5 years and, therefore, not included in the table.

For the October-December rainfall, the longer-period oscillations ( $>30$ years) 


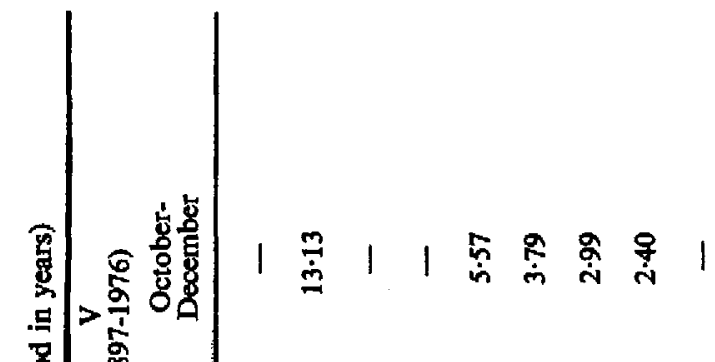

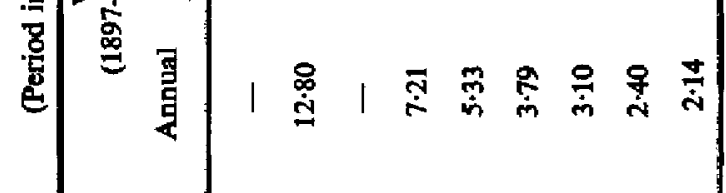

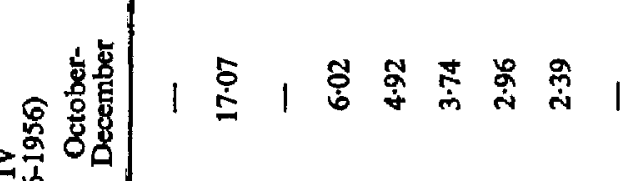

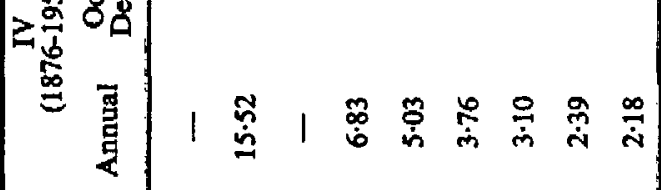

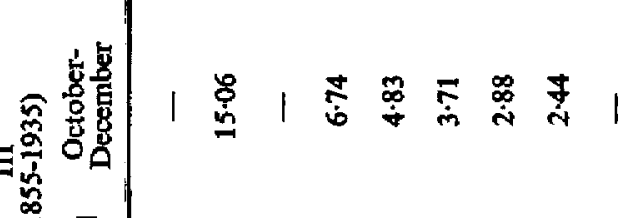

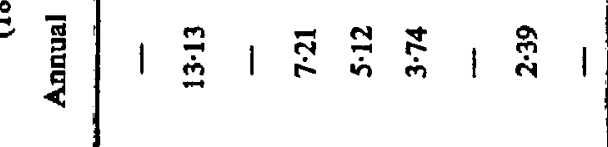

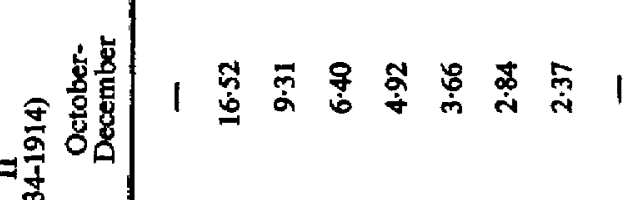

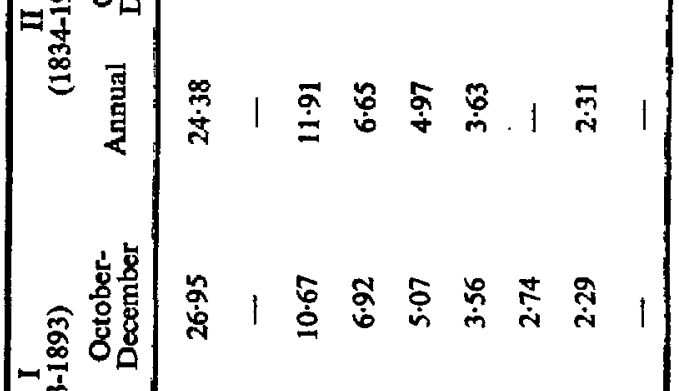

$$
\begin{aligned}
& \text { 咅 }
\end{aligned}
$$


are absent. While the amplitudes of the oscillations in the period range of 13 to 27 years are quite large for the subsets, the actual frequency is not stationary. In contrast, in the higher frequency range (period $<5$ years) significant oscillations common to all the subsets occur with periods around 5.1, 3.7, 2.9 and 2.4 years.

When we consider the annual rainfall, we note that here again no periodicity in excess of 30 years is seen for any of the blocks and the peaks between 10 and 25 years are not stationary. There is no evidence of any periodicity corresponding to the solar cycle oscillation of nearly 11 years, except for the spectral lines detected in the first two blocks spanning the years 1813-1913. But here again the two periods differ substantially (10.7 and 11.9 years respectively). Thus the spectral peak at 11.38 years noticed in the total length of data is only an artifact of the data sample and not a stable feature that can establish an association between solar activity and rainfall. The common periods to all the 5 subsets are again in the higher frequency range with periodicities corresponding to around $6.9,3.7$ and 2.4 years.

\section{Analysis of monthly rainfall data}

Since analysis of data with annual values could give information on periods only in excess of 2 years $(0.5 \mathrm{c} / \mathrm{y}$ being the Nyquist frequency), we next considered time series of the rainfall data with data interval of 1 month specially to identify the nature of the biennial oscillation in the rainfall at Madras. Taking 30-year span at a time and considering 10 blocks with 15-year overlap between successive blocks we computed the spectrum using a low value of $L(\simeq 10 \%)$ so that the spectrum would be smooth without undue line-splitting, separately for each block. The spectral amplitudes at each frequency from the 10 samples were then combined to yield a stacked spectrum wherein only those lines which were repetitive in most of the blocks will be

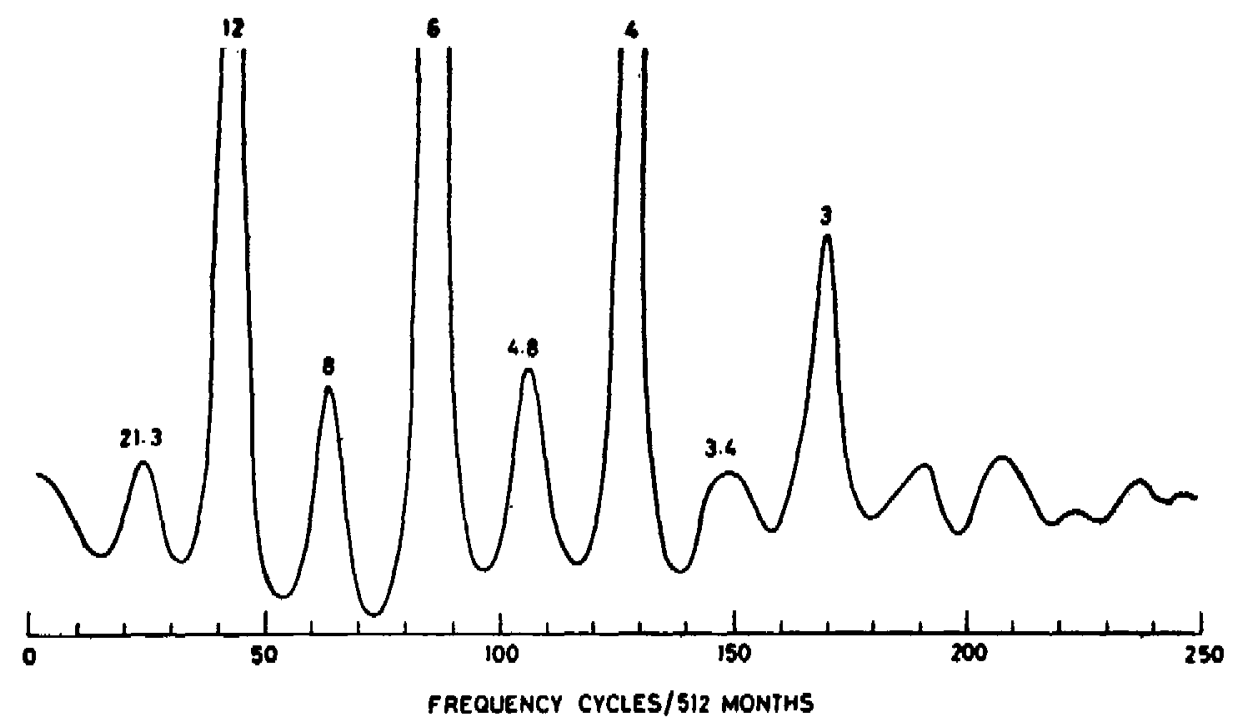

Figure 2. Stacked spectrum of monthly rainfall at Madras. Ordinate is amplitude in arbitrary units. Period (months) of spectral peaks are indicated. 
prominent while others with variable frequencies are likely to be averged out. The stacked spectrum of monthly rainfall at Madras is shown in figure 2 .

The spectrum is dominated by a very strong annual line and its harmonics at 6,4 and 3 months. The only other spectral peaks of significance are at 8 and 4.8 months. These two could be sidebands caused by amplitude modulation of the 6 months' wave by a biennial ( 24 months) pulse. Alternatively they could also be the harmonics of the biennial oscillation. However, the nearest period to the biennial oscillation in the spectrum is a feeble 21.3 month line. It is also significant that while the QBO is present when annual values were considered for analysis the same is absent in the monthly data. This could be mainly due to the fact that the phase of this oscillation in NE and SW monsoon rainfall are differing from each other by nearly $140^{\circ}$ and tend to cancel each other. Phase angles at each frequency were computed in the Fourier spectra of the time series.

\section{Acknowledgement}

The authors are thankful to Prof. B N Bhargava for going through the manuscript and for suggestions.

\section{References}

Blackman R B and Tukey J W 1959 The measurement of power spectra (New York: Dover) Burg J P 1975 Maximum entropy spectral analysis Ph.D. Thesis Stanford University. California Chen W Y and Stegen G H 1974 J. Geophys. Res. 793019

Cooley J W and Tukey J W 1965 Math. Comput. 19297

Courtillot V, Le Mouel J L and Mayaud P N 1977 J. Geophys. Res. 822641

Currie R G 1975 J. Atmos. Terr. Phys. 37439

Fougere P F, Zawalick E J and Radoski H R 1976 Phys. Earth Planet. Interiors 12201

Lacoss R T 1971 Geophysics 36661

Radoski H R, Fougere P F and Zawalick E J 1975 J. Geophys. Res. 80619

Rangarajan G K and Arora B R 1977 J. Atmos. Terr. Phys. 39641

Rangarajan G K and Bhargava B N 1974 Proc. Indian Acad. Sci. 80 A 249

Rao K N and Raghavendra V K 1973 Met. Monograph No. 7 IMD Poona

Smylie D E, Clarke G K C and Ulrych T J 1973 Methods in computational physics (New York: Academic Press) 13391

Ulrych T J 1972 J. Geophys. Res. 771396

Ulrych T J and Bishop T N 1975 Rev. Geophys. Space Phys. 13183 